\title{
Report: EWM panel discussion on gender balance in mathematics at the European Congress of Mathematics
}

Eugénie Hunsicker

A European Women in Mathematics-sponsored online panel on Gender Balance in Mathematics took place on 22 June, 2021 as part of the ECM. As a long-time activist and advocate for diversity in mathematics, I was delighted to have been asked to chair the panel. The panelists were also a group of committed diversity advocates in a range of leadership roles within our community:

- Dr. Shabnam Beheshti (Director of Education, Department of Mathematics, Queen Mary University of London and researcher in mathematical relativity),

- Prof. Klavdija Kutnar (8ECM Organizing Committee Deputy Chair, Professor of Mathematics and Rector of University of Primorska, and researcher in algebraic graph theory),

- Prof. Volker Mehrmann (president of EMS, and researcher in numerical analysis),

- Prof. Jill Pipher (past-president of AMS, and researcher in harmonic analysis and PDE),

- Prof. Carola-Bibiane Schönlieb (former EWM convenor, and researcher in applied PDE, inverse problems and mathematical imaging).

The topics we discussed in the panel covered the span of issues of policy, practice and culture that research suggests are relevant for driving the gender-biased outcomes we observe. These include topics such as recruitment, promotion and the impact of family commitments, but also structural barriers in the shape of mathematical careers and cultural biases in the ways that success is judged within mathematics - topics that we need to discuss in our community if we are to make further progress. I hope the ideas presented in the panel encourage further discussion.

CHAIR: Tell us about the current status of gender equity in mathematics in your country. Where has there been progress over the past 10 years? Where are the biggest challenges remaining?

SHABNAM: One of the most significant improvements I have seen in the UK is the recognition that decisions about policies and practices have measurable consequences in terms of diversity, and that relevant data should be analysed and benchmarked by discipline to understand this. There needs to be a broader awareness of the value of these analyses in getting people on board at the policy level.
However, although the number of women in senior levels is increasing in the UK, it isn't happening rapidly enough. One problem is that underrepresented groups are under tremendous pressure to be visible and mentor others, which can be in direct tension with the promotion and progression of the mentors because of the way we place value for promotion on different criteria and activities. Another factor is that people on teaching-based versus research-based contracts get siloed away from each other, which is unhelpful.

KLAVDIJA: I am from Slovenia, where there is gender balance in terms of students studying mathematics at bachelors and masters level. The situation begins to skew towards men at PhD level, and is even worse for women employed in mathematics departments. At my university, one quarter of department members are female; the proportion is even lower at other universities in Slovenia. There are only four female full professors in the whole country; this is progress - three of these full professors were promoted in the past 10 years! Also in the mathematics, physics and astronomy society in Slovenia, we have established an active committee for women. The biggest challenge is that we have quite a few women with excellent research records who have the title of Assistant Professor but only the salary of a teaching associate.

VOLKER: In Germany, political policies have been set so that the gender balance in mathematics should in principle increase drastically and quickly, through rules about gender bias and inviting people to interview. However, the number of women professors has not improved very much. This is mainly because the number of female applicants is still very low - likely due to the terrible timeline in hiring. Professors are usually hired when they are between 35 and 45 years old. Before that, you must keep moving between temporary positions, which is particularly difficult for women with families. Another challenge is that if you follow the rules on committee representation strictly, the few women professors we have are overburdened with committee work. So, although the national policies are good, given the hiring situation at universities, I am not too optimistic that gender equality will improve quickly. 
In the EMS, the executive committee is now $40 \%$ female, and together with the Women in Mathematics Committee, we have come up with an explicit plan to substantially increase female representation in editorial boards over the next few years. The or ganising committees for this congress have also done quite a good job with invited speakers, continuing the trend towards improvement of recent years. However, we are still in a bad situation with respect to the prize winners, where equity has not improved. This is something where I don't have a clue what to do. We can certainly make sure that more women sit in the organising and prize committees, but that would be another burden on them, so there is a catch.

JILL: I want to start by saying this is a really timely moment for discussion of these issues. There is already a fair amount of research that shows that the effects of the pandemic are especially hard on women in science. In fact, a recent article in Nature showed that submissions by male authors to the arXiv increased during the pandemic ${ }^{1}$, unlike submissions by women.

To talk about the situation in the US, I am going to back up a bit. Nationally, in 1977, $13 \%$ of mathematics PhDs were awarded to women. By 1993 , this had climbed to $28 \%$, so real progress was made over this time. Since then, it increased to $33 \%$ in the early 2000s, but now the numbers are again at $29 \%$ - in the past 30 years there has been really no progress. Why is that? We know that work/life issues have a greater effect on women. The career timeline in the US, starting from the $\mathrm{PhD}$, is that you are expected in about 7 years to reach a tenured or permanent position, and these are the same years during which women are creating families as well as careers. Even the adoption of gender-neutral family leave practices in academia has been shown in some discip-

${ }^{1}$ nature.com/articles/d41586-020-01294-9 lines to privilege men². As Dean Alison Davis-Blake in Michigan, puts it, "Giving birth is not a gender-neutral event." Finally, a 2020 National Academy of Sciences report ${ }^{3}$, had a really sobering conclusion: bias, discrimination, and harassment are major drivers of underrepresentation. I think it is really difficult for well-intentioned, caring people in our profession to face such a conclusion, but face it we must, or I feel that we'll make no further progress.

CAROLA: Since I moved to the UK about 10 years ago, many actions in favour of women mathematicians have emerged in the UK, such as the national Athena Swan Charter. Many student societies have also popped up. Moreover, hiring started to change so that search committees actively encourage female candidates they want to apply. So, the situation has changed in terms of actions and awareness, but there is certainly not equity yet. There is a long way to go, particularly the higher you go up in the profession, and this starts already at $\mathrm{PhD}$ level. One of the biggest challenges is that family and housework are not considered equal endeavours between two people in a couple.

On the European level, I am the former Convenor of the EWM, and we have AWM in the US. The fact that these associations still exist and are super active is a sign that there is still a lot of work to do and that young female mathematicians are looking for networks to exchange experiences. When Elena Resmerita and I were active as Deputy Convenor and Convenor, we also tried to collect gender ratios in mathematics by countries, which was a sobering exercise ${ }^{4}$.

\footnotetext{
2 iza.org/publications/dp/9904

${ }^{3}$ nap.edu/read/24994/chapter/1

${ }^{4}$ For statistics from 2005, see womenandmath.wordpress.com/pastactivities/statistics-on-women-in-mathematics/
}

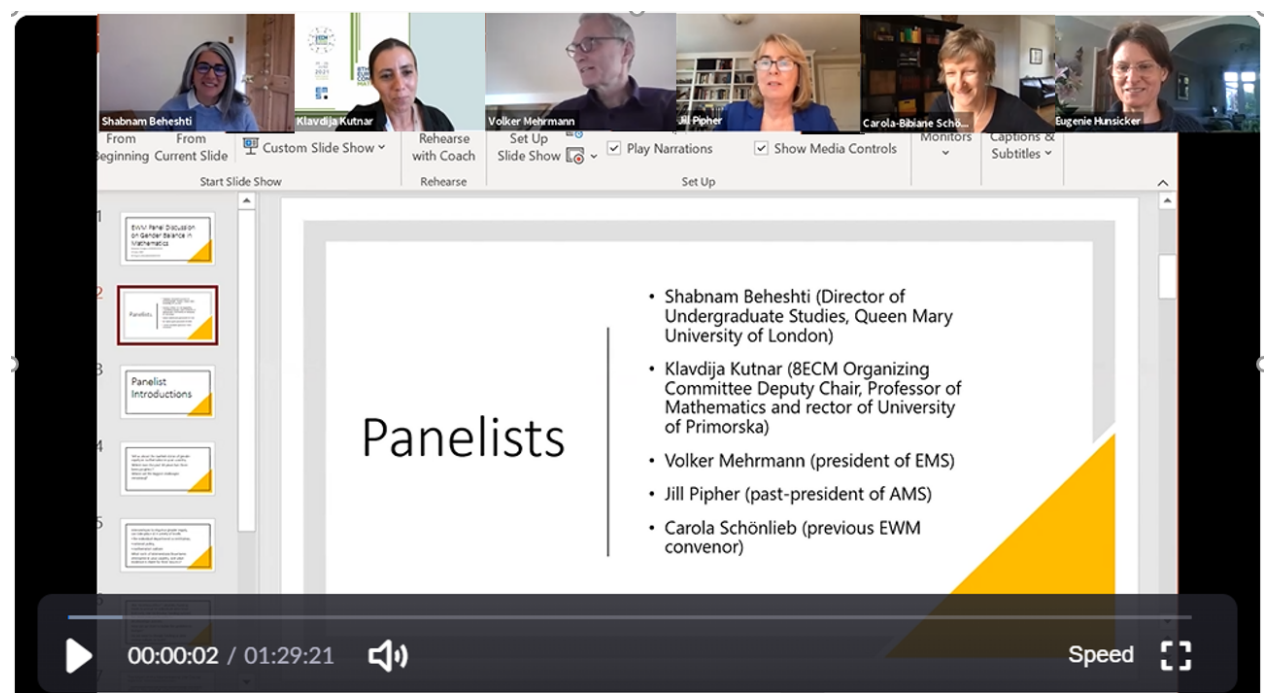

From left to right, panellists Shabnam Beheshti, Klavdija Kutnar, Volker Mehrmann, Jill Pipher, Carola-Bibiane Schönleib, and panel chair, Eugénie Hunsicker. 
CHAIR: Interventions to improve gender equity can take place at a variety of levels: at the individual department or institution, in national policy, or in mathematics culture. What sorts of interventions have been attempted in your country, and what evidence is there for their success?

SHABNAM: At the individual level, it is the right time for a conversation on intersectionality between gender issues and issues around decolonisation of the curriculum. For example, even if the development of quantum mechanics was influenced directly by the works of Bohr and Schrödinger, there are many people on the planet now who are working in the field who don't "look like them". Our students need to see dotted lines of work going into the future and see themselves as participating in a diverse, living discipline.

At the department level, what we have done well, and which has become easier now with online teaching, is to allow schedules to accommodate personal lives. Also, be aware of administrative allocations being made with a gender bias, such as putting women primarily in teaching-related roles, which are then not valued as highly in promotion applications. This is a place where just looking at data helps.

At a university or professional society level, ring-fence funding to help researchers keep their research going after a particular gap, not just childcare; this may also help address two-body problems Learned societies have already helped to tackle this. For example, I was a recipient of the LMS Grace Chisholm Young fellowship, which supports mathematicians who have had to take a break from formal employment for any of a variety of reasons. As a recipient of this Fellowship, I was able to establish myself as a mathematician and find a new position after relocating to the UK for family reasons. Finally, professional and learned societies also have a role to play in advocacy and data gathering for policy-making at national level.

KLAVDIJA: Individual departments are critical. I reached my position of Rector because the mathematics department at my university supported me, first to the level of Dean, then to the level of Rector. I would like to see other universities in Slovenia follow suit. I am now involved in making national policies, for example concerning national scientific awards. We don't have enough women candidates to select a balanced set of awardees between women and men as we are required by law to do. Also, we are currently preparing a new act for research and innovation, involving several committees at national level, which again must be gender-balanced. The policies are in place - departments now need to recognise female researchers and suggest them for these awards and positions.

VOLKER: At the institutional level, we have to give stronger support to the lower levels in the leaky pipeline. For example, we are organising days for girls at our university, to get more girls interested in studying mathematics and science. Then we need departments and universities to support women at the PhD level so they have a stronger track record when they apply for positions, for instance with extra grants so that they can go on research trips and so on, I think that has a positive effect. We have tried this in Berlin.

In terms of national policy, it has had a positive effect that about $9 \%$ of the budget of each university depends on meeting gender balance quotas, which is an incentive to hire women. The countereffect is that universities that focus primarily on the humanities have greater success in reaching this goal than technical universities that focus primarily on science and engineering subjects.

JILL: In terms of mathematics culture, I want to focus a little on the stereotypical assumptions about mathematics careers and mathematicians. There are a lot of studies that show that the perception that women are not as gifted or talented at maths, and that this is what is required to succeed, is very damaging. We also know from many studies that role models matter. This causes its own problems, however, because the same senior women are called on over and over to serve as role models, which hurts their careers.

Like Klavdija, I have been strongly supported by my department in obtaining leadership roles in my department and university. The experience of being supported is phenomenally important.

Concerning strategies at a policy level, I think that we need leaders who acknowledge the importance of addressing the problem of gender underrepresentation and resources that are allocated to address equity issues, such as for recruitment, teaching, training, creating transparency, collecting data, and so on. Actions like recognising implicit bias and reading and writing letters of recommendation with an eye for gendered language all require effort and attention and training. Mathematicians, like all academics, need reminding that this is necessary and important and they need support for doing it.

CARolA: As both Klavdija and Volker mentioned, you can't give prizes to women if they aren't nominated. It is crucial to work on increasing the number of nominations of women mathematicians for the prizes of national, European, and international societies, which need to be active in encouraging this. The LMS is very aware of this and has really improved things. On the European level, both the EMS Women in Mathematics Committee and the EWM are working hard to encourage nominations of strong women mathematicians. The AWM also has a prize committee that makes similar efforts, as many societies should.

SHABNAM: I would like to raise here the topic of mentorship. People tell me, "You need a mentor to do X," and I say, "I don't need a mentor. What I need is an advocate who is in the room when people are making decisions and who will stand up for the quality of my work." Mentorship has its time and place, but I wonder if we have to move from local practice to policy when we are talking about people who are in permanent positions but don't progress. 
VOLKER: I fully agree about the importance of advocates. Being in committees all the time, I see that people advocate for their own students and field, but not so much for young women in other fields. If you are lucky, you get enough nominations of women for prizes, and even better, a few EMS prizes to women. We need to appeal to the community to think beyond subdisciplines, and to work on looking for the best women to whom to award prizes, independently of field, rather than focusing on the fifteenth person in line in your own subarea.

JILL: I couldn't agree more that there are policy issues and that mentorship will not solve everything. The AMS prize oversight committee was founded particularly to accomplish this - to look at our processes and develop ways to arrive at a more diverse set of prize winners. There are undoubtedly things that occur in all processes that we don't see if we don't look at the data that come out of them. We need to take a look at the data on outcomes for prizes, hiring, promotion and so on and think about whom we are leaving out.

CHAIR: There are reports coming out now that focus on mid-career as a place where there is an issue ${ }^{5}$. For instance, in the UK, the pipeline in mathematics is roughly $23 \%$ women from $\mathrm{PhD}$ up to professor, then it plummets to $13 \%$ among professors. There is a tendency to focus policy and support on people early in the pipeline, to try to get it right for the future, while forgetting those further down the pipeline. We need to think about how to help people who are struggling now wherever they are, rather than always trying to start over to get it right with the next generation.

CHAIR: The "Matthew Effect", whereby funding tends to accrue to individuals who have had early and continuous funding success, has been seen to disadvantage women especially. How can we start to tackle this problem in Europe? Do we need to change funding or peer review culture, or both?

SHABNAM: There is certainly the idea that "success begets success". I would propose that funding agencies allow more people to keep their research ticking over to keep the mathematical community alive, instead of devoting large amounts of funding to only a few individuals, who are then supposed to become leaders in their field. I am very curious to see what uptake you would get in terms of gender balance if instead of having half-a-million-pound grants, they were divided by four and funded more people.

\footnotetext{
${ }^{5}$ chronicle.com/article/the-associate-professor-trap; advance-he.ac.uk/ knowledge-hub/mid-career-academic-women-strategies-choices-andmotivation-final-report-activity

${ }^{6} \mathrm{dfg}$.de/download/pdf/dfg_im_profil/geschaeftsstelle/publikationen/ studien/studie_gender_effects.pdf; pnas.org/content/115/19/4887
}

KLAVDIJA: I am very familiar with the concrete implications of the Matthew Effect. It took me quite a while to be successful in obtaining funding for a project at the Slovenian Research agency. I was finally awarded a grant three years ago, the first maths project led by a female.

For Europe, I think the funding situation will change because, as you probably know, every institution that wants to apply for EU grants needs to prepare an action plan to improve gender balance and equal opportunities. Universities are therefore forced to consider this problem at the institutional level in order to apply for EU grants.

VOLKER: The European Research Council (ERC) is a wonderful example of the Matthew Effect. The money is going to the strongest individuals and only to those. I think it is also very common in many countries. This is not primarily about gender. We have a saying in Germany, "The devil always shits on the biggest pile". This is the statement that describes the situation very well. I see a colleague at the panel from the ERC online, and maybe she can say something.

MARIA GONZALEZ (ERC): I am a woman mathematician, but I left mathematics research and for the past seven years have been a scientific officer for the ERC working on panel coordination. Based on my observations, I believe that culture is the main thing you have to change. There are certain beliefs about what pure or applied mathematicians do or what statisticians do, and sometimes the mathematics community forgets that you are a single community that should be working together rather than fighting with each other.

Some of the problems you mention are built into the culture, such as desired trajectories: if someone is a speaker here or there, or publishes here or there, it gives them a certain status. In the past 2-3 years, we have had more women on panels, and I have heard more discussion about different ways to evaluate applications and candidates. I think this is a good sign. I believe in working from the inside.

At the ERC, it is the panel [of mathematicians] that decides on the funding - if there is such a thing as the Matthew Effect, it is coming from something in the culture of the community. But I also think the ERC and the community are jointly responsible for spotting these problems and then working to improve them. Maybe I am overly optimistic, but I think if we start with you, the community of mathematicians, and we forget about fighting among our subcommunities and forget about our biases and accept this as a common responsibility, then things may change.

CHAIR: We hear the same comments in the UK from the EPSRC Mathematical Sciences panel: members of the mathematical community are the ones making the decisions. But I think there is also confusion in the community about to what extent and how we can change the way we do reviewing and run panels. We have 


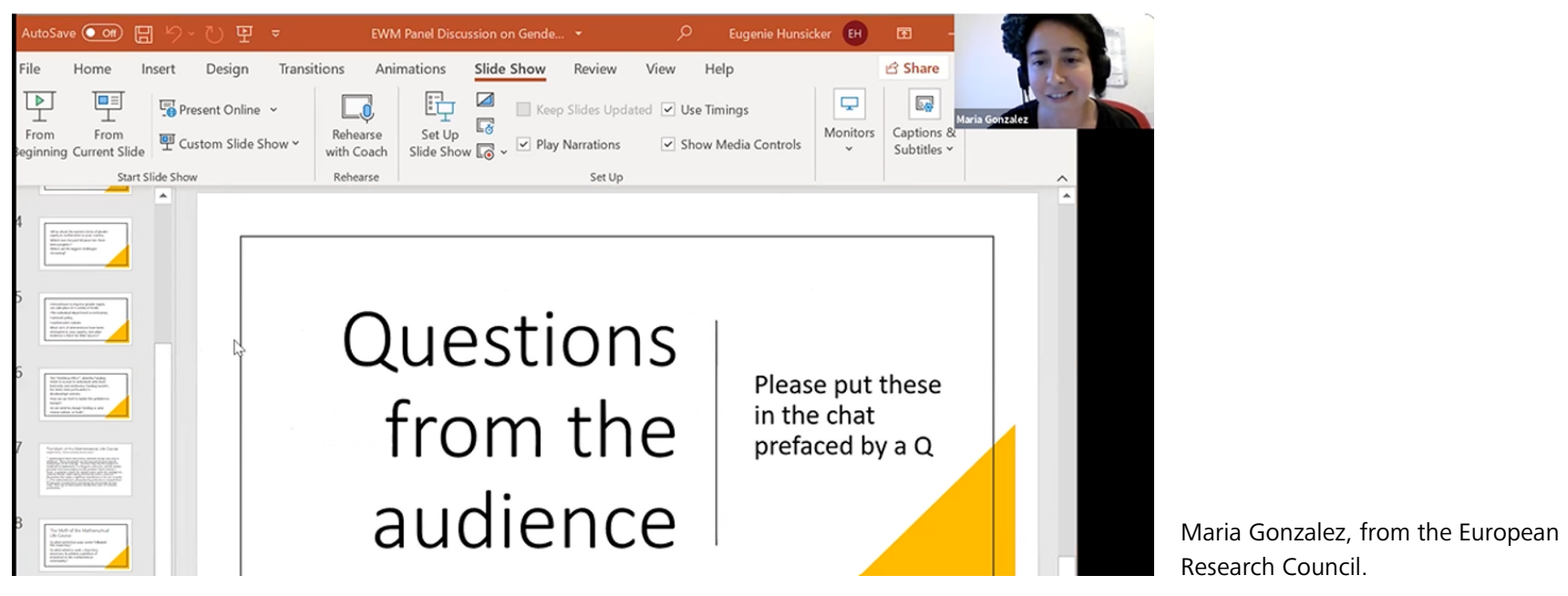

always done it in a certain way, which we assume is okay because we have always done it that way. If we do it in a new way, is that okay, or will we go against some agency policy? How members of the mathematics community can shape the way that the funding policies are enacted is an important question.

JILL: In the US, the majority of funding for mathematics comes from the NSF, and within the NSF, the division of mathematical sciences competes for its funding with other sciences. So the pressure to fund large and new initiatives and award big money to specific places is tremendous. However, the Division of Mathematical Sciences (DMS) devotes a significant part of its budget to funding 6 or 7 mathematics institutes in the country, and through these institutes, it is possible to disperse smaller awards to many, many mathematicians, bringing them to programs, conferences and workshops. I think the institute program within the DMS has been a remarkably successful one, especially since the number of institutes has expanded from three in 1999 to over twice that now.

In terms of peer review, I think that even in situations where it seems difficult or unlikely to succeed, we should really consider double-blind review, not just for review of grants, but also for articles. The argument against this is that people put everything on the arXiv and therefore reviewers know who the author is within a few pages. But I would say that even so, if your first impression is the mathematics, not the person, not where they are from, this creates a different place to start. Even if you know after five pages who the author is, still you started in a different place.

CAROLA: The Matthew Effect is to some degree related to the way that your track record will influence your future opportunities, which exists in all professions and is not a totally bad thing. But it does cause problems for people whose career path is not standard and, for example, start their careers later than usual. I think we need to change reviewing procedures and how we are educating reviewers to take account of nonstandard careers.

CHAIR: In "Women Becoming Mathematicians", Margaret Murray" talks about "The Myth of the Mathematical Life Course", which is characterised by early recognition of talent and an uninterrupted path to professional success. To what extent has your career followed this trajectory? To what extent is such a trajectory necessary to achieve a position of eminence in the mathematical community?

SHABNAM: My career has not followed this trajectory in any way, shape or form. But here's the important thing. Even though it hasn't, on paper I can make it look like it has, which has been important for successfully navigating through each next stage in my career. I've come to the realisation, from the comfort of a permanent position, that the further my trajectory moved from the "standard one", the harder I had to fight to retain my identify as a mathematician. It wasn't that I had difficulty being a mathematician, but rather I had difficulty staking my own claim on what a mathematician looked like.

CAROLA: Early on, when I started a PhD, things went really, really badly. I got very discouraged and almost quit. I would have done so, if our head of department hadn't said, this isn't about you or your skills, it is just the wrong situation for you. Leave, go somewhere else. I lost quite a bit of time. But from the time I started my PhD again in the right community, I have been really lucky, and things have gone very well.

VOLKER: Let me be a little provocative. I haven't followed this trajectory, but I am a man and an applied mathematician. I am not a gold medallist of the mathematical Olympiad, and I haven't been

\footnotetext{
7 ams.org/notices/200107/rev-green.pdf
} 
in the career hot-spot driving seat, as many of the colleagues we see [at the Congress] are. So maybe we should get rid of the Olympiads and prizes altogether in order to avoid having this hotspot-straighton-career idea still drive what we do in mathematics.

CHAIR: I am fully in favour of being provocative! This is a question about the culture - how do we change the cultural expectations? What you implied is that it may not be necessary to follow this trajectory to become a mathematician, but most of those who have become prize winners of eminence have done so. This suggests that when we select people to nominate for prizes, the expectation is that nominees will have had careers like this, which is a very gendered trajectory. So maybe we also need to change what we are looking for in prize winners.

JILL: The notion that mathematicians are somehow born to do this or must be on a track on an early age keeps a lot of people out of the profession. I can point to a lot of well-known and successful people who have not been on this trajectory. I was just at a reception for the Joan Birman Fellowship for mid-career women that she endowed at the AMS. She got her BSC in 1948, and her PhD in 1968: a very different trajectory, followed by a fantastic mathematician. We really do have to do something about this cultural construct that there is a single path to success in mathematics.

Eugénie Hunsicker is a Reader in Mathematics working at Loughborough University in the UK. After studying mathematics at Haverford College, she received her PhD in Mathematics from the University of Chicago, in the US. She has recently finished six years as the Chair of the London Mathematical Society's Women and Diversity in Mathematics Committee, and she is current Chair of the Athena Forum, an organisation of STEMM professional societies in the UK collaborating on equity, diversity and inclusion (EDI) in UK academia, hosted by the Royal Society. She has also recently been appointed as Honorary Officer for Equality, Diversity and Inclusion for the Royal Statistical Society, and has advised a wide range of professional and other organisations on EDI matters.

e.hunsicker@lboro.ac.uk

\section{Advanced Studies: Euro-Tbilisi Mathematical Journal}

Advanced Studies: Euro-Tbilisi Mathematical Journal is the continuation of the Tbilisi Mathematical Journal founded in 2008. Under this new name the journal was relaunched in September 2021 to reflect the increase of its standards and broadening of its coverage.

It is a fully refereed mathematical journal accepting original high-quality research papers in all areas of pure and applied mathematics. It belongs to the Tbilisi Centre for Mathematical Sciences and it is supported by the Georgian National Academy of Sciences and the European Mathematical Society.

The journal is electronically handled by Project Euclid of Cornell University Library and Duke University Press.

Editor-in-Chief:

Hvedri Inassaridze (Tbilisi State University)

High quality submissions in all areas of pure and applied mathematics are strongly invited.

http://tcms.org.ge/Journals/ASETMJ

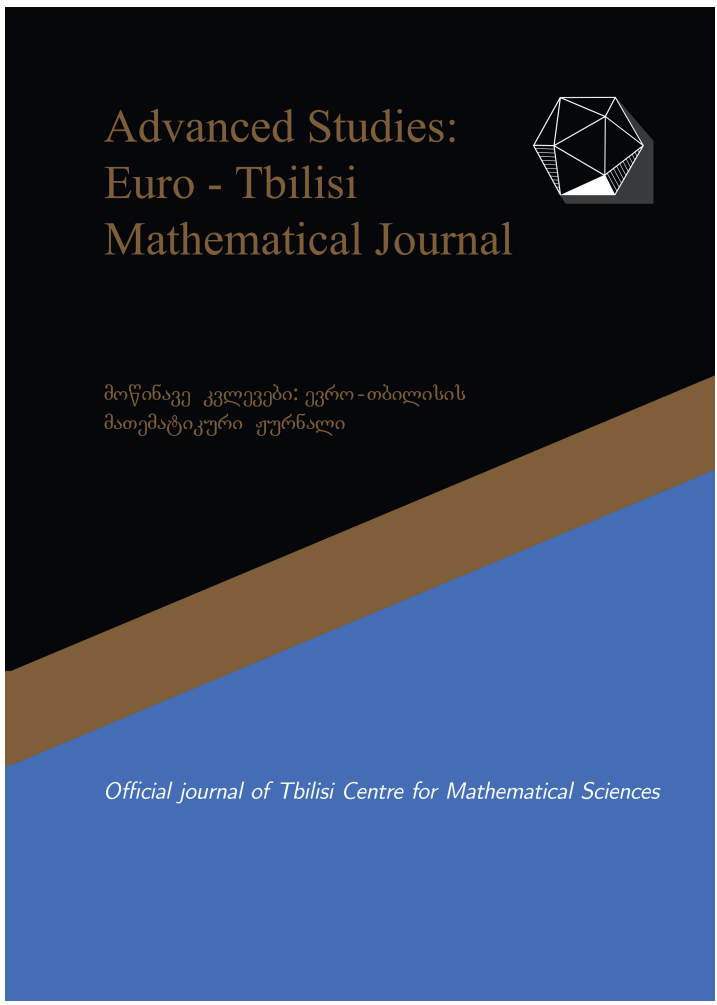

